\title{
Reflets
}

Revue ontaroise d'intervention sociale et communautaire

\section{L'empowerment et intervention familiale : concept paradoxal occultant parfois la pauvreté}

\section{Marie Drolet}

Volume 3, numéro 1, printemps 1997

Enfance et familles en contexte d'appauvrissement

URI : https://id.erudit.org/iderudit/026151ar

DOI : https://doi.org/10.7202/026151ar

Aller au sommaire du numéro

Éditeur(s)

Reflets : Revue ontaroise d'intervention sociale et communautaire

ISSN

1203-4576 (imprimé)

1712-8498 (numérique)

Découvrir la revue

Citer cet article

Drolet, M. (1997). L'empowerment et intervention familiale : concept paradoxal occultant parfois la pauvreté. Reflets, 3(1), 55-79.

https://doi.org/10.7202/026151ar
Résumé de l'article

Les compressions budgétaires dans les services sociaux, d'éducation et d'appuis à la famille sont omniprésentes. À l'hiver 1996, le gouvernement Harris annonçait une restructurationéventuelle des services de santé mentale à l'enfance fondée sur l'efficience ciblée. On mise surune intervention précoce, brève, intensive et efficace. L'empowerment des individus et desfamilles semble une voie à explorer. Cet article vise à cerner cette notion et ses divers courants,puis à l'appliquer à l'intervention familiale individualisée selon une perspective écologique.Sans en faire une analyse approfondie, nous saisirons au départ les énoncés ministériels parrapport à la pauvreté et au travail avec les familles. L'empowerment occulte-t-il alors unesur-responsabilisation individuelle des problèmes? Banaliser ce concept permet-il de nier lapauvreté et son impact sur des familles?
Tous droits réservés (C Reflets : Revue ontaroise d'intervention sociale et communautaire, 1997
Ce document est protégé par la loi sur le droit d'auteur. L'utilisation des services d’Érudit (y compris la reproduction) est assujettie à sa politique d'utilisation que vous pouvez consulter en ligne.

https://apropos.erudit.org/fr/usagers/politique-dutilisation/ 


\title{
L'empowerment et intervention familiale : concept paradoxal occultant parfois la pauvreté
}

Les compressions budgétaires dans les services sociaux, d'éducation et d'appuis à la famille sont omniprésentes. A l'hiver 1996, le gouvernement Harris annonçait une restructuration éventuelle des services de santé mentale à l'enfance fondée sur l'efficience ciblée. On mise sur une intervention précoce, brève, intensive et efficace. L'empowerment des individus et des familles semble une voie à explorer. Cet article vise à cerner cette notion et ses divers courants, puis à l'appliquer à l'intervention familiale individualisée selon une perspective écologique. Sans en faire une analyse approfondie, nous saisirons au départ les énoncés ministériels par rapport à la pauvreté et au travail avec les familles. L'empowerment occulte-t-il alors une sur-responsabilisation individuelle des problèmes? Banaliser ce concept permet-il de nier la pauvreté et son impact sur des familles?

\author{
Marie Drolet ${ }^{1}$ \\ École de service social, Université d'Ottawa
}

Il est devenu un lieu commun de mentionner les compressions budgétaires aux plans fédéral et provinciaux dans le domaine des services sociaux, municipaux, de la santé et de l'éducation. En plus des coupures déjà réalisées aux niveaux de l'aide sociale et de divers services d'appui aux familles, le gouvernement de Mike Harris annonçait, à l'hiver 1996, une restructuration éventuelle des services de santé mentale à l'enfance. Cette reconfiguration s'effectuera en ciblant l'efficience: la compression des dépenses, la restriction des services à des clientèles priorisées, la maximisation des ressources, la coordination régionale et des indicateurs de performance en fonction de résultats mesurables (Association 
ontarienne des centres de santé mentale pour enfants 1996). Outre les services mandatés par la Loi sur les jeunes contrevenants et la Loi sur les services à l'enfance et à la famille, les services à l'enfance, dits d'investissement, devront prévenir chez les enfants à hauts risques le recours à des services subséquents. On mise sur une intervention précoce, ciblée, brève, intensive et efficace, offerte dans les familles, à l'école, dans et avec les communautés (Association ontarienne des centres de santé mentale pour enfants 1996). Seraient ainsi évités des services ultérieurs plus intrusifs et plus coûteux, tels les placements résidentiels; on vise donc à intervenir dès l'émergence du besoin et plus particulièrement avant l'âge de 6 ans.

Malgré un intérêt pour le bien-être, la santé et le développement de tous les enfants et de tous les jeunes, la reconfiguration ministérielle vise spécialement les enfants victimes ou à hauts risques de négligence et de maltraitance, ceux aux besoins émotifs exceptionnels, ou ceux ayant des troubles de comportement majeurs ou des conduites déviantes. Il importe de noter que les investissements à l'égard des services sociaux doivent aussi résulter de façon efficace en une meilleure sécurité et une protection adéquate pour le public.

Ces énoncés n'annoncent-ils pas une intervention sociale limitée aux familles exigeant un contrôle social et légal très étroit en raison des hauts risques pour la protection des enfants, des jeunes et de la collectivité? Cette notion étroite de "risques» n'occulte-t-elle pas notamment ceux associés à la pauvreté? De

"...la privatisation grandissante des services sociaux et d'aide à la famille ne limitera-t-elle pas les clientes des services gratuits, subventionnés par l'État, aux familles pauvres?" surcroît, la privatisation grandissante des services sociaux et d'aide à la famille ne limitera-t-elle pas les clientes des services gratuits, subventionnés par l'État, aux familles pauvres? Suite à cette restructuration des agences consacrées à la santé mentale des enfants, le partenariat avec la communauté, avec ses ressources informelles et formelles, se limitera-t-il à leur utilisation pour compenser les coupures de services? Or, malgré cette conjoncture, «la profession du service social doit continuer à intervenir au nom des plus démunis, tout en sensibilisant le public, entre autres, aux effets nuisibles des politiques gouvernementales mesquines» (Andreae, 1996: 3). 
«Parler d'empowerment serviraitil selon cet angle à surresponsabiliser des individus face à leurs problèmes? Serait-ce un concept permettant parfois de nier la pauvreté et son impact sur des individus et des familles?"
Dans ce contexte, une voie semble apparaitre : l'empowerment des individus, des familles et des communautés. Cet article cherche à cerner la notion d'empowerment et ses divers courants, puis à l'appliquer à l'intervention familiale individualisée, selon une perspective écologique. Sans en faire une analyse approfondie, nous sonderons, au départ, les énoncés ministériels ontariens, pour mieux saisir comment l'intervention avec les familles qu'on y propose occulte la pauvreté. Parler d'empowerment servirait-il à sur-responsabiliser des individus face à leurs problèmes? Seraitce un concept permettant parfois de nier la pauvreté et son impact sur des individus et des familles? À l'encontre de ce courant technocratique, cet article considère que l'empowerment, à tout le moins, contextualise les problèmes individuels et familiaux de même que l'intervention familiale individualisée. Celle-ci ouvre la voie pour que les clientes exercent un sentiment de contrôle sur leur réalité : un empowerment psychologique ouvrant la voie à une participation collective, à une défense de droits d'accès à des ressources.

\section{Énoncés ministériels, intervention familiale et pauvreté}

\section{Des familles à hauts risques}

Les clientèles désignées pour les services de santé mentale à l'enfance qu'énumèrent les énoncés ministériels limitent l'intervention de ces agences à des contextes de protection sociale. La plupart des services ciblés coïncident avec ceux offerts aux familles identifiées à hauts risques par les services américains de soutien aux familles (Family preservation services) (Kaplan et Girard 1994). Cette pratique dite novatrice mise sur l'intervention réalisée à domicile et de concert avec les ressources de la communauté dans le but de maintenir les enfants dans leurs familles (Home-based practice) (Hodges et Blythe 1992). Ces dernières présenteraient un potentiel élevé de crises perpétuelles; or, une 
"Le seuil de faible revenu désigne les niveaux de revenu brut où on consacre $56,2 \%$ ou plus aux dépenses de première nécessité: nourriture, logement et vêtements.» combinaison complexe d'interactions difficiles à l'intérieur de la famille et de conditions de vie environnementales limitatives pointe des facteurs précipitants en perpétuelle sourdine (Hodges et Blythe 1992). À travers tout ce prisme écologique, il y a la pauvreté.

Ne doit-on pas ici se remémorer qui la pauvreté concerne? Le "seuil de faible revenu» désigne les niveaux de revenu brut où on consacre $56,2 \%$ ou plus aux dépenses de première nécessité : nourriture, logement et vêtements (Conseil national du bienêtre social 1996:4). Pour une région urbaine de 500000 habitants et plus comme Ottawa-Carleton, le seuil de pauvreté est de 20981 \$ pour une famille de deux personnes, de 26670 \$ pour une famille de trois, de 30708 \$ pour une famille de quatre. Sans tenir compte du nombre de personnes dans la famille ou le ménage, $52 \%$ des francophones de la région métropolitaine gagneraient moins de 30000 \$ (Statistique Canada, cité dans Malenfant et Delisle 1996). D'ailleurs, une forte proportion de la population franco-ontarienne vivrait sous le seuil de pauvreté (Savard, cité dans Diallo et Reitsma-Street 1995). En guise d'exemple, au Centre psycho-social pour enfants et familles d'Ottawa-Carleton, $53 \%$ des familles ayant fait une nouvelle demande de counselling entre mars 1996 et janvier 1997 ont un revenu annuel de $22000 \$$ et moins. De toutes ces demandes, $53 \%$ provenaient de mères vivant seules avec des enfants. Ces familles dirigées par une femme sont certes un des deux groupes les plus pauvres au Canada. En 1994, près de 60 \% d'entre elles vivaient sous le seuil de pauvreté. Pour les jeunes mères de moins de 25 ans, le taux de pauvreté grimpe jusqu'à près de $90 \%$; enfin, pour les mères ayant des enfants de moins de 7 ans, le taux de pauvreté atteint $83 \%$ (Conseil national du bien-être social 1996: 1).

Il importe ici d'attirer l'attention sur une mise en garde souvent oubliée: toutes les familles pauvres ne requièrent pas de l'aide institutionnelle, et toutes les familles nécessitant une protection pour leurs enfants ou leurs jeunes ne sont pas pauvres (Ronnau et Marlow 1993). Sans être un facteur exclusif expliquant des problèmes individuels et familiaux, la pauvreté contribue souvent à leur émergence et constitue un facteur de hauts risques notable 
"Sans être un facteur exclusif expliquant des problèmes individuels et familiaux, la pauvreté contribue souvent à leur émergence et constitue un facteur de hauts risques notable et un stresseur constant important.» et un stresseur constant important (Garbarino et al. 1986; Gelles 1993). La pauvreté a été maintes fois identifiée comme un facteur de risques pour la maltraitance et surtout pour la négligence des enfants et des jeunes (Garbarino et al. 1986; Dore 1993; Gelles 1993; Trocmé 1994; Trocmé et al. 1995). Ces risques s’accroissent encore davantage quand la famille vit isolée. Plus précisément, Trocmé et al. (1995) rapportent qu'en Ontario, au moins $38 \%$ des cas d'enfants maltraités en 1993 provenaient de familles vivant de prestations d'aide sociale. Cette étude souligne aussi que $48 \%$ des enquêtes de maltraitance portaient sur les mères et celles-ci étaient responsables de $82 \%$ des cas de négligence. Or, près de $80 \%$ des familles monoparentales sont dirigées par des femmes. Comme les chiffres précédents le démontrent, la plupart de ces familles monoparentales vivent dans la pauvreté. Le retrait des pères, la lourde charge et la pauvreté qu'affrontent ces femmes engendrent un stress constant, souvent un isolement.

La pauvreté apparaît également comme un des éléments-clé pour cerner les troubles de comportement et la déviance (Vitaro et al. 1994), deux autres problématiques importantes pour les énoncés ministériels. La pauvreté est aussi vue comme un déterminant majeur au placement d'enfants en familles d'accueil (Lindsay cité dans Boudreault et al. 1994). Il faut de nouveau se rappeler qu'en plus de toutes ces considérations sur les familles susceptibles de nécessiter de l'aide ou une protection pour leurs enfants, la privatisation grandissante des services sociaux et d'aide à la famille limiterait les clientes des services gratuits et des agences institutionnelles, subventionnés par l'État, presque exclusivement aux familles pauvres. Pourtant, les énoncés ministériels l'occultent totalement pour cibler des problématiques.

\section{Des interventions familiales individualisées}

Par ailleurs, en plus de cibler des problématiques à privilégier, l'énoncé ministériel décrit ci-haut jette un éclairage sur ses 
"On mise sur une intervention précoce, brève, intensive et efficace, ciblée à l'aide d'objectifs concrets, offerte dans les familles, à l'école, dans et avec les communautés. Or, cette intervention s'apparente aux programmes de sauvegarde de la famille mis sur pied aux Etats-Unis pour éviter le placement des enfants et pour les maintenir dans leurs familles.» intentions quant à l'intervention d'investissement. On mise sur une intervention précoce, brève, intensive et efficace, ciblée à l'aide d'objectifs concrets, offerte dans les familles, à l'école, dans et avec les communautés. Or, cette intervention s'apparente aux programmes de sauvegarde de la famille mis sur pied aux ÉtatsUnis pour éviter le placement des enfants et pour les maintenir dans leurs familles (Kinney et al. 1990; Carrier et al. 1992; Dagenais et Bouchard 1993; Boudreault et al. 1994; Kaplan et Girard 1994). Ces programmes s'appliquent aussi avec des nuances aux adolescents et adolescentes en difficulté (Odesse et al. 1991). Ils se centrent sur le soutien à la famille, sur la consolidation de ses forces, sur sa propre prise en charge de ses difficultés, sur des apprentissages d'habiletés et le renforcement du réseau social (informel et formel). Ils se basent dans la famille et la communauté, établissant une coupure avec une distribution de services dans les agences. Ainsi ces programmes de soutien intensif aux familles «visent trois objectifs principaux : assurer la sécurité du jeune et de sa famille, améliorer le fonctionnement de la famille et tenter en dernier recours d'éviter un placement non nécessaire» (Dagenais et Bouchard 1993: 505).

De fait, ces programmes de maintien des familles se fondent sur l'utilisation positive de la crise, ici familiale. Certes, le danger demeure que les crises soient successives et même perpétuelles, que la sécurité de l'enfant soit compromise, surtout lors de grande pauvreté ou de toxicomanie (Cole 1995). Or l'énoncé ministériel vise également à éviter de telles récidives et des circonstances exigeant des services plus intrusifs et plus coûteux (voire à éviter le placement), en intervenant dès l'émergence du besoin. «En revanche, une intervention qui ciblerait la pauvreté (véritable facteur de hauts risques) finirait par remettre en question les structures socio-économiques de notre société. C'est bien plus simple de tenir les mères responsables» (Trocmé 1994: 26). Prétendre devenir centré sur la famille pour les services aux enfants deviendrait alors un enjeu crucial de lutte contre la pauvreté (Cole 1995). Sinon, une intervention au bienfait des enfants, prévenant certains abus et consolidant vraiment les familles demande au moins des programmes d'insertion et de soutien 
communautaires: aide concrète, groupe de parentage, halteaccueil et actions collectives d'entraide (Cole 1995).

Pour les familles vivant dans la pauvreté, Gutiérrez et al. (1995) et Pinderhughes (1995) prônent de surcroît une intervention axée sur l'empowerment des familles, ici défini comme au minimum une appropriation de son pouvoir sur sa réalité. Kissman et Allen (1993) avancent aussi une intervention fondée sur l'empowerment pour les mères seules, surtout en contexte de pauvreté et d'isolement; elles suggèrent une contextualisation, une démystification de la mère seule jusqu'à contrecarrer l'étiquette négative de la famille brisée construite sur la famille nucléaire traditionnelle. Axé sur une démarche intériorisée et sur les compétences, l'empowerment invite ici les familles à s'approprier leur vie et à chercher un soutien social. Mais surtout, de telles actions s'insèrent à travers une collectivation des situations individuelles et familiales. Par ailleurs, Freedman (1996) considère l'empowerment comme un élément-clé, sous son angle collectif, comme une défense de droits et une lutte contre la pauvreté.

\section{Émpowerment : tendances et définition}

"Pire encore, au nom de la capacité des personnes à se prendre en charge, on essaie souvent de justifier la réduction ou même la suppression des services auxquels elles ont droit.»
Depuis près de 10 ans, le concept d'empowerment suscite de plus en plus d'engouement (Adams 1996; Le Bossé 1996). En contrepartie, cette vitalité provoque parfois un usage abusif de ce concept qui en dilue l'impact (Breton 1994 a et b;Adams 1996), allant jusqu'à créer de la confusion (Perkins et Zimmerman 1995; Dallaire et Chamberland à paraître). Pire encore, «au nom de la capacité des personnes à se prendre en charge, on essaie souvent de justifier la réduction ou même la suppression des services auxquels elles ont droit» (Le Bossé 1996:128). En guise d'exemple, l'énoncé de principe ci-après, prononcé au Québec par un centre régional de décisions gouvernementales, en illustre une utilisation réductionniste et technocratique, qui s'éloigne de sa définition:

Des personnes au coeur de nos pensées et de nos actions: 
«L'empowerment se définit comme un processus par lequel une personne développe le sentiment d'exercer un plus grand contrôle sur sa réalité par des actions concrètes dans des conditions de vie incapacitantes.»

\section{(...) Pour cela, il faut entre autres favoriser des attitudes} qui visent à aider et à accompagner les personnes et les groupes pour leur permettre de développer leurs compétences et leurs habiletés à la mesure de leurs capacités dans un esprit d'empowerment, c'est-à-dire d'être apte à se prendre en charge, et non de culpabilisation (Régie régionale de la santé et des services sociaux Mauricie-Bois-Francs, 1996: 2.2).

Quoique prononcé au Québec, cet énoncé de l'empowerment va aisément dans la lignée ministérielle ontarienne, soit: la prise en charge individuelle des problèmes, une négation de leur contextualisation et de la pauvreté, de même qu'une surresponsabilisation familiale.

Par contre, quel que soit le courant représenté, certains éléments suscitent un consensus. L'empowerment se définit comme un processus par lequel une personne développe le sentiment d'exercer un plus grand contrôle sur sa réalité par des actions concrètes dans des conditions de vie incapacitantes (Le Bossé 1995). Ces conditions réferent à la pauvreté, à des problèmes personnels majeurs, à la marginalité, mais aussi à l'exclusion et à l'oppression. L'empowerment vise à la fois les personnes, les groupes et les communautés; il s'agit d'un sentiment d'exercer ou l'exercice même d'un contrôle réel sur les événements qui concernent les personnes impliquées (Rappaport 1987), et non de leur unique prise en charge. L'empowerment devient donc une stratégie d'intervention libératrice des rôles ou des places sociales sans ou avec peu de pouvoir (Pinderhughes 1995); on parle aussi de démarches individuelles et collectives pour un accès plus équitable aux ressources du système social, de défense de droits des plus démunis (Le Bossé 1996).

La traduction littérale du verbe empower par le Webster lui offre trois sens : avoir autorité sur quelqu'un, habiliter une personne à faire ou avoir plein pouvoir pour réaliser. Ces trois traductions jettent un éclairage sur les diverses tendances du concept d'empowerment, dont le processus repose sur la notion de pouvoir. Le pouvoir, "c'est de transformer un choix en une décision» 
"La dimension

fondamentale $d u$ pouvoir devient alors le contrôle, un contrôle véritable. L'autonomie apparaît comme le fait d'échapper au contrôle des autres et d'avoir une emprise sur sa réalité.»
(Lemieux dans Ninacs 1995: 76). Cette perception du pouvoir repose sur des capacités néanmoins existantes de choisir, puis de décider en fonction de ce choix et d'agir à partir de cette décision. Selon une perspective davantage structurelle, ici féministe, le pouvoir demande d'être inséré dans le contexte global de la société, à travers les rapports sociaux inégaux. Schelgel (citée dans Dagenais 1985) associe le pouvoir à la capacité de contrôler sa personne, sa réalité, ses activités, et même parfois celles des autres. La dimension fondamentale du pouvoir devient alors le contrôle, un contrôle véritable. L'autonomie apparait comme le fait d'échapper au contrôle des autres et d'avoir une emprise sur sa réalité. Le pouvoir décisionnel se confond parfois à l'autorité, socialement reconnue et légitimée, qui peut "prendre des décisions concernant autrui (...), et ce par opposition au pouvoir d'influence qui, lui, peut être exercé aussi bien par des individus dénués d'autorité formelle» (Dagenais 1985: 109).

Certaines nuances à la notion d'empowerment découlent de ces visions du pouvoir. Un courant plus écologique pointera des rapports sociaux injustes et inéquitables, l'impact des macrosystèmes sur les individus et les familles, leur intériorisation de la culture discriminante, une vision contextualisée, sociale et critique des problèmes individuels et familiaux, une réappropriation du pouvoir individuel et collectif par une participation à des organismes communautaires (Zimmerman 1995; Le Bossé 1996). La tendance la plus radicale inscrit plutôt le processus d'empowerment dans une démarche de libération des rapports d'oppression, un pouvoir formel et un rapport de force en faveur des exclus de notre société, à qui on a même évacué le pouvoir d'influence (Breton 1994a; Gutiérrez 1994; Lee 1994). L'empowerment devient en soi politique (Adams 1996). Bref, un continuum se dessine d'un angle technocratique jusqu' à une position radicale (Parsloe 1995;Vincent 1996).

La psychologie communautaire considère le phénomène de l'empowerment comme son champ d'intérêt principal, sa finalité, sa marque idéologique, sa compréhension écologique (Rappaport 1987 dans Le Bossé et Lavallée 1993). Cette discipline a contribué à mieux saisir l'impact sur le développement d'une personne 
"...l'empowerment se fonde sur les capacités de la personne et sur ses solutions, sur des démarches individuelles, de groupe et d'action communautaire pour avoir accès à des ressources, pour consolider son insertion sociale et détenir une emprise, voire un contrôle sur sa réalité.» engendré par sa participation à des groupes communautaires (Kieffer 1984; Zimmerman et Rappaport 1988; Le Bossé 1995; Perkins et Zimmerman 1995;Zimmerman 1995). Cette discipline a exploré l'empowerment psychologique, ici défini lors de l'application de l'empowerment à l'intervention familiale individualisée.

Le travail social se définit aussi comme une profession encourageant l'empowerment (Parsons 1991; DuBois et Miley 1996). Simon (1994) en perçoit des dimensions à chaque étape de l'histoire du travail social américain, surtout en action communautaire. Cette intervention a jusqu'à présent particulièrement collaboré à la prise d'une place sociale par les femmes, les minorités visibles, les gais et lesbiennes (Lee 1994; Gutiérrez et al. 1995). S'inscrivant dans chaque méthode en service social, sous diverses approches, l'empowerment se fonde sur les capacités de la personne et sur ses solutions, sur des démarches individuelles, de groupe et d'action communautaire pour avoir accès à des ressources, pour consolider son insertion sociale et détenir une emprise, voire un contrôle sur sa réalité (Ninacs 1995). Cela «se retrouve à la fois comme approche, processus et finalité partout où des intervenants et intervenantes tentent d'appuyer les efforts autonomes des individus et des collectivités pour se tailler une place à part égale et à part entière sur l'échiquier social» (Ninacs 1995: 69). Cet appel à une société plus juste va dans le sens de «contribuer à modifier durablement les conditions (lire sociales) qui ont présidé à l'émergence du problème» (Le Bossé, 1996: 138).

Par ailleurs, les différences entre les deux courants principaux qui définissent l'empowerment (la version technocratique étant ici exclue) ne correspondent pas à des disciplines, des modes d'intervention, des clientèles ou des objectifs. Cette différence réside dans des grilles d'analyse sociale divergentes. Pour le premier courant à tendance écologique, la réflexion se base sur «l'inégalité du partage des ressources entre ceux qui ont les moyens de se faire entendre et ceux qui n'ont pas droit au chapitre» (Le Bossé 1996: 137). Pour le second, plus radical, la pratique avec les personnes pauvres ou les membres de groupes opprimés se fonde sur une vision historique de l'oppression construite sur des 
"Cette pratique

élaborée sur une

conscientisation

politique réaffirme le

potentiel inhérent à

chaque personne, réitère que le privé est politique, qualifie de nouveau l'oppression de destructrice et victimisante, conduit à l'action visant le changement social.» structures sociales érigées sur des rapports de sexe, de race, d'ethnie, d'orientation sexuelle et finalement de classe. Cette pratique élaborée sur une conscientisation politique réaffirme le potentiel inhérent à chaque personne, réitère que le privé est politique, qualifie de nouveau l'oppression de destructrice et victimisante, conduit à l'action visant le changement social (Breton 1994a; Gutiérrez1994; Lee 1994). Au fil de tous ces courants se construit le continuum de grilles d'analyse et d'intervention (Parsleo 1995).

Malgré ces différences, des ponts ou des consensus s'érigent parfois. À titre d'exemple, une étude empirique menée par Lorraine Gutiérrez (1995), tenante d'une démarche politique, met en lumière que les intervenantes considèrent l'empowerment en terme de processus psychologique et de changement individuel. Par ailleurs, aider les clientes et les familles dans leurs diversités à survivre aux réalités en mouvance interpelle en soi l'empowerment (Pinderhughes 1995). L'intervention d'empowerment, axée surtout sur la consolidation des réseaux et mise de l'avant par Dunst et al. (1994), vise essentiellement une adaptation sociale; or, ses modalités d'intervention correspondent sous plusieurs angles à celles de l'approche communautaire familiale en CLSC (Centre local de services communautaires) telle que définie au Québec par Pauline Gingras (1992). L'approche structurelle fondée aussi sur l'empowerment et élaborée notamment par Maurice Moreau (1989; 1993; Lee 1994; Lévesque et Panet-Raymond 1994) donne également des pistes d'action: un regard collectif et des actions individualisées. L'intervention brève axée sur la solution (De Shazer cité dans Simard et Turcotte 1992) apparait comme une voie complémentaire et une opérationnalisation de certaines assises, soit la cliente compétente, actrice de ses solutions et de ses stratégies. Par ailleurs, J. Rappaport $(1993 ; 1995)$ incite à considérer la thérapie narrative comme une avenue à investiguer auprès des individus pour appuyer une démarche de changement fondée sur l'empowerment.

En service social, Breton (1994) ainsi que Mullender et Ward (1991) ont documenté les aspects de l'intervention de groupe favorisant l'empowerment; Ninacs (1995) en a résumé divers aspects en lien avec l'action communautaire; Dallaire et Chamberland (à 
"L'entraide et l'action sociale mises de l'avant par les autres méthodes en travail social nous semblent complémentaires, voire essentielles à une démarche individualisée.» paraître) ont mené une réflexion sur des interventions de prévention-promotion d'habiletés sociales. En définitive, divers éléments de sa relation dynamique avec la participation communautaire ont été étudiés, et ce également en psychologie communautaire; par contre, son application à l'intervention familiale individualisée a été plutôt délaissée.

Tout en s'inscrivant dans une perspective écologique qui interpelle l'approche de réseaux, une meilleure adaptation sociale et davantage un empowerment psychologique, la section qui suit suggérera des éléments instrumentaux de l'approche structurelle à une intervention familiale individualisée d'empowerment. Cette intégration se veut un apport proactif, voire paradoxal, dans la restructuration des services de santé mentale à l'enfance et de ses agences. Nous utiliserons des pistes de Dunst et al. (1994) pour une perspective d'adaptation sociale, des éléments issus de l'approche structurelle (Moreau 1989; Moreau et al. 1993; Lee 1994; Lévesque et Panet-Raymond 1994; Guttiérrez et al. 1995) et certains éléments de l'empowerment psychologique pouvant offrir des voies de soutien (Zimmerman et al. 1992;Zimmerman 1995). L'entraide et l'action sociale mises de l'avant par les autres méthodes en travail social nous semblent complémentaires, voire essentielles à une démarche individualisée. Bref, il s'agit d'un bilan exploratoire d'éléments consensuels entre les courants et les pistes à valider.

\section{Empowerment et intervention familiale individualisée}

Puisqu'il s'agit essentiellement d'un processus fondé sur les capacités des personnes, sur leurs solutions et sur l'exercice réel d'un contrôle croissant sur leur réalité, une intervenante ne développe pas l'empowerment de ses clientes; elle offre des conditions facilitant l'empowerment, elle l'éveille ou le suscite. Les clientes s'habilitent elles-mêmes en accomplissant des actions concrètes. En effet, elles choisissent les actes à poser en fonction de leur 
"Puisqu'il s'agit essentiellement d'un processus fondé sur les capacités des personnes, sur leurs solutions et sur l'exercice réel d'un contrôle croissant sur leur réalité, une intervenante ne développe pas l'empowerment de ses clientes; elle offre des conditions facilitant l'empowerment, elle l'éveille ou le suscite." cadre de référence et du contexte social, pour ainsi voir leurs conditions de vie modifiées en leur faveur, pour avoir de l'emprise, se réaliser, et finalement détenir une place sociale. Certes, cette démarche exige du temps: il faut que l'action et ses réalisations deviennent des éléments de renforcement grâce à la rétroaction et à des prises de conscience (LeBossé et Lavallée 1993). Nous sommes donc très loin des préoccupations technocratiques de prises en charge rapides et efficaces des clientes par elles-mêmes, faisant la promotion d'interventions prescrites dès l'émergence du problème afin d'éviter d'autres services ultérieurs, plus intrusifs et plus coûteux. Certes, l'empowerment vient ici en contradiction avec sa récupération technocratique. Les intervenantes et même les agences faciliteront l'émergence d'interstices en faveur des droits des clientes, d'une considération de la pauvreté. Des prises de position seront-elles opportunes?

L'empowerment se veut par définition un processus fondamentalement démocratique où intervenantes ou chercheures se voient assigner un rôle d'accompagnatrice (Fetterman et al. 1996). En relation d'aide individualisée, familiale, de groupe et communautaire, la relation cliente/intervenante se construit sur une relation égalitaire : partage des connaissances, décisions de la cliente pour elle-même, plein pouvoir ou du moins respect de celle-ci quant à l'action à mener, partage des limites et des biais personnels et institutionnels (Moreau 1989; Lee 1994; Ninacs 1995; Le Bossé 1996).

L'empowerment psychologique peut par ailleurs pointer vers des axes d'intervention. Celui-ci se distingue tout d'abord de l'empowerment individuel qui ne contextualise ni la personne, ni la famille, ni la démarche dans la réalité sociale et ses enjeux pour l'empowerment purement individuel (qui s'apparente à la vision technocratique); les limites et les embûches deviennent synonymes de problèmes personnels. Sont alors perdues la vision écologique, la dynamique personne en situation et l'interaction famille et environnement (Zimmerman 1990). Pour mieux mesurer ou évaluer un processus favorisant l'empowerment psychologique, ce concept comporte trois aspects: l'intrapersonnel, l'interactionnel et le comportemental (Zimmerman et al. 1992; Zimmerman 1995). 
Le niveau intrapersonnel réfere à ce que les personnes pensent de leur propre capacité d'influencer leur situation et leurs difficultés actuelles, leur quotidien, leur environnement immédiat, leur tissu social, ainsi que les systèmes sociaux et politiques. Ce niveau concerne:

- le contrôle tel que perçu par la personne concernée (et son désir de contrôle);

- une perception de la maîtrise sur son environnement (le lieu ou foyer de contrôle);

- un recadrage positif des difficultés ou de la situation (aspects ou zones de sa vie);

- une recherche des solutions entreprises et des exceptions au problème exploré;

- une perception de sa compétence et de son efficacité (sentiment de compétence);

- la motivation d'exercer ce contrôle (la possibilité et les opportunités à entrevoir);

- un travail en faveur de l'estime de soi et de la fierté.

L'aspect interactionnel correspond aux possibilités pour une personne de négocier avec succès:

- sa capacité d'explorer de multiples besoins à divers niveaux (une vision idéale);

- sa connaissance de maintes ressources selon plusieurs besoins (une taxonomie);

- sa priorisation sur des besoins de survie de base à partir de ses forces et limites;

- sa capacité de prendre des décisions éclairées à partir de faits et de ses émotions;

- sa capacité de résoudre des problèmes, d'organiser ou de mettre en place des solutions;

- sa capacité d'obtenir des ressources considérées prioritaires et de l'aide concrète;

- une connaissance, une analyse sociale et critique de son environnement. 
La mise en action de cette dimension interactionnelle tient également compte d'aspects familiaux :

- le contrôle tel que perçu par les divers membres de la famille, l'exercice du pouvoir;

- une description du fonctionnement familial (tensions, forces et essais de solutions);

- une observation partagée des scénarios (patterns) interactionnels et relationnels;

- une appropriation du changement interne en fonction de l'écoute et du respect;

- un partage des préoccupations, sentiments, désirs d'entraide mutuelle et des loisirs;

- une consolidation maximale des liens avec les réseaux informels et formels.

Le comportemental désigne des actions spécifiques et des habiletés techniques à solidifier :

- qui permettent de s'adapter de façon proactive aux difficultés de la vie courante;

- qui donnent de l'emprise et un contrôle sur la réalité quotidienne et sociale. (Kieffer 1984; Zimmerman et Rappaport 1988; Simard et Turcotte 1992; Zimmerman et al. 1992; Dunst et al. 1994; Le Bossé 1995; Zimmerman 1995; Zimmerman dans Fetterman et al. 1996).

Gutiérrez et al. (1995) ont exploré entre autres l'empowerment psychologique à l'aide de propos d'intervenantes sociales. Ce concept est ici défini à la fois comme un but pour la pratique auprès d'individus et de familles (effets escomptés) et comme une démarche de travail avec des clientes: un processus et des résultats. Quatre mots ressortent : contrôle, confiance, pouvoir et choix.

Pour avoir non seulement le sentiment d'exercer un contrôle, mais aussi la capacité d'influencer sa réalité, il importe d'agir concrètement afin de contrôler les résultats d'une action. Ce choix se fonde sur le monde, les préoccupations et les valeurs de la cliente, sur la perspective de la famille. Il s'agit d'atteindre des objectifs réalisables et graduels, aux effets concrets, visibles, 
«Il faut donc identifier avec soin les endroits d'incapacités ou d'impuissance, les besoins concrets de survie de base, les priorités accessibles.

Ces réussites alimentent l'estime de soi, la confiance en soi, la fierté, le courage et l'espoir.» palpables, mesurables, perceptibles ou du moins nommables, et ce à partir de ses forces, capacités, compétences ou réalisations précédentes. Il faut donc identifier avec soin les endroits d'incapacités ou d'impuissance, les besoins concrets de survie de base, les priorités accessibles. Ces réussites alimentent l'estime de soi, la confiance en soi, la fierté, le courage et l'espoir. Parfois, ces petits pas et la confiance qu'ils engendrent, cette image positive de soi sont les seuls éléments d'ancrage et d'emprise dans une situation difficile, dans un contexte d'appauvrissement. Ne peuvent-ils pas redonner assez d'estime de soi pour tenter une autre action, briser l'isolement, nourrir son tissu social, aller vers des lieux communautaires?

L'exploration des choix et la prise de décisions sont aussi des gestes importants auprès des clientes pour qui un tel processus est inhabituel dans des agences, des adolescentes ou des jeunes mères monoparentales et pauvres, par exemple. Cette démarche alimentera l'estime de soi et la fierté au lieu d'une perception de soi victimisante qui amène souvent à se blâmer, à ne voir que ses propres torts et à être envahie par des sentiments négatifs et pessimistes. Or, cette victimisation est accentuée par la survalorisation des éléments individuels d'une difficulté et de ses variables psychologiques qui entrent en jeu contre les clientes et minimisent l'importance des causes sociales. Une écoute attentive notamment des émotions suscitées, leur contextualisation et leur validation sociale facilitent l'émergence de pistes d'emprise et d'actions possibles dans l'esprit de la cliente. La collectivisation des problèmes individuels et la matérialisation des problèmes sociaux chez des individus et des familles donneront une force à l'atteinte de cibles accessibles, aidant à aller au-delà de l'intériorisation (Moreau1993; Lee 1994).

Cette analyse critique recadre; elle redonne du pouvoir aux clientes, car elle nomme les enjeux individuels, familiaux et sociaux (Lévesque et Panet-Raymond 1994). Elle renomme les agents stresseurs liés à la pauvreté et leurs impacts sur la famille; bref, cette analyse sociale contextualise. Partager des observations sur le fonctionnement familial ne nie pas les compétences et les solutions de cette famille ni de ses membres; ceci devient un 
«...l'empowerment

supporte le

développement d'un sens interne d'être soi, il rend capable de se mettre en action et de risquer des nouveautés à partir de cette intériorité; il nourrit de fait une congruence.» partage de connaissances, un recadrage, une reformulation, une universalisation, une insertion dans un construit social. Ce reflet devient une occasion pour la famille de changer des manières d'agir nuisibles: une réappropriation et une conscience des rapports de pouvoir et de violence. Cette collectivisation offre aussi une ouverture pour rejoindre d'autres clientes dans la même condition ou dans des situations comparables: un rappel à l'entraide et à l'action communautaire, un bris de l'isolement et de la victimisation, une consolidation des acquis et du tissu social, un pouvoir sur un contexte de pauvreté, une amélioration des conditions concrètes.

L'empowerment agira ainsi sur la sensation de vulnérabilité, sur les pensées négatives intrusives et l'anxiété (Ozer et Bandura 1990). En effet, l'empowerment supporte le développement d'un sens interne d'être soi, il rend capable de se mettre en action et de risquer des nouveautés à partir de cette intériorité; il nourrit de fait une congruence. Cette conscience de soi construite à travers une compréhension sociale et une prise de la parole aide à une telle réflexion sur soi; cette consolidation peut motiver une personne à s'engager encore plus dans sa propre vie face à soi-même, parfois face aux autres (famille, tissu social et communauté), et à devenir actrice de sa vie (Shields 1995). Cette force lui rendra alors davantage possible l'établissement de choix, fondés sur une diversité de possibilités et élaborés selon une stratégie éclairée de résolution de problèmes et de prises de décision. Or, «développer la conscience et l'accès à multiples choix est identifié comme un élément crucial de l'empowerment» (Gutiérrez et al. 1995: 538). De surcroît, l'empowerment est associé à l'autonomie. Cette fois-ci, l'autonomie correspond à l'indépendance des usagères, à leur liberté eu égard à leur vie. Cette perspective ne contredit cependant pas celle qui la définit comme le fait d'échapper au contrôle des autres et d'avoir une emprise sur sa réalité. Dans une perspective écologique ou structurelle de l'empowerment, il est important de ne pas négliger le besoin de soutien des clientes (Gutiérrez et al. 1995). Il ne s'agit pas d'une sur-responsabilisation individuelle, mais d'un appel en faveur de cette force en soi. 
"Cette démarche augmentera en effet l'autosuffisance de la famille, l'estime de soi de ses membres, leur compétence et leur sentiment de contrôle sur leur réalité.»
L'empowerment des familles tel qu'il est conçu par Dunst et al. (1994) porte un regard particulier sur le renforcement du réseau social. Supporter, renforcer et consolider les familles s'insèrent dans un processus d'empowerment où la démarche de mobilisation de ressources externes reçoit une attention prépondérante en vue d'une auto-prise en charge. Cet apprentissage applicable à d'autres occasions devient ainsi un apport important. Considérant les clientes comme les premières compétentes, le plan de mobilisation se construit à partir de l'identification des besoins réalisée par les clientes et leurs familles. Mettant l'accent sur le soutien et les capacités d'aller chercher les ressources, une consolidation des forces familiales permet cette mobilisation des ressources internes pour rejoindre celles externes à la fois informelles et formelles. Cette démarche augmentera en effet l'autosuffisance de la famille, l'estime de soi de ses membres, leur compétence et leur sentiment de contrôle sur leur réalité. Elle soutient une insertion communautaire.

\section{Conclusion : pour éviter la banalisation de l'empowerment}

En dépit des grilles d'analyse divergentes sur la notion de pouvoir, son accès et la structure sociale, l'empowerment se conçoit néanmoins en premier lieu comme une philosophie, une idéologie et une vision faisant la promotion des compétences des personnes et des familles, et ce en vue d'une société plus juste et équitable. L'empowerment devient ainsi un processus, un partenariat, une démarche de travail avec des clientes fondée essentiellement sur la mise en valeur de la compétence des clientes, sur une relation égalitaire et une auto-prise en charge des actions concrètes sur son quotidien ou sa réalité. Ce concept vise des buts pour la pratique sociale auprès d'individus et de familles. Sont ainsi ciblés des effets escomptés : un sentiment de prise de contrôle ou un exercice du pouvoir par des actions concrètes. Sous l'angle 
psychologique, cette démarche collabore notamment à l'amélioration de l'estime de soi, de sentiments de compétence et d'habiletés de résolutions de problèmes. Une perspective communautaire de l'empowerment psychologique soutient une conscience culturelle, sociale et collective, de même qu'une pensée critique. Le réseau social de soutien en est solidifié (Rappaport 1987; Dunst et al. 1994; Gutiérrez et al. 1995; Ninacs 1995; Zimmerman 1995). Une participation communautaire accrue devient une étape subséquente. Bref, l'empowerment qui contextualise devient une voie de solution.

Certains visent uniquement une adaptation sociale: ils encouragent alors personnes et familles à dépasser les barrières de la pauvreté et à devenir des membres actifs de la société. Ils supportent leurs compétences pour valoriser une force personnelle, une entraide familiale et un soutien social. Mettant l'accent sur les solutions et non sur les problèmes, on passe rapidement à l'action; on tente d'éviter la récidive, le besoin d'autres services et une intrusion accrue (Dunst et al. 1994). L'accès inéquitable aux ressources sociales devient la cible primaire de l'intervention; or la pauvreté peut alors y être plus mise en veilleuse.

«S'engage alors une recherche active d'options pour réaliser des rêves et pour nourrir un espoir réaliste à l'aide d'améliorations concrètes des conditions objectives de vie. Outre cette démarche, l'approche structurelle s'inscrit clairement dans une défense des droits des clientes.»
Par contre, Moreau $(1989 ; 1993)$ et Lee (1994) invitent, au cours d'une démarche d'empowerment individualisée ou familiale, à créer un pont et à entrer dans le monde des clientes : s'amorce alors dans le respect une restructuration cognitive dévictimisante, libératrice, mobilisante, accrue par le contact avec d'autres comme soi. La pauvreté ne s'avère pas occultée. Au contraire, la collectivisation des problèmes individuels et familiaux, de même que cette matérialisation des enjeux sociaux deviennent source d'un sentiment de prise de contrôle sur sa réalité et d'une conscience sociale critique. S'engage alors une recherche active d'options pour réaliser des rêves et pour nourrir un espoir réaliste à l'aide d'améliorations concrètes des conditions objectives de vie. Outre cette démarche, l'approche structurelle s'inscrit clairement dans une défense des droits des clientes. Or, la lutte contre la pauvreté, surtout dans la réorganisation sociale actuelle, remet sur le tapis la défense des droits. 


\section{«L'empowerment} appliqué à l'intervention familiale individualisée perd vraiment une partie fondamentale de son sens s'il ne débouche pas sur de telles activités collectives d'entraide, une participation communautaire et des solidarités.»
En terme de soutien aux familles vivant dans la pauvreté,le sondage mené par le Conseil de planification sociale d'Ottawa-Carleton (Malenfant et Delisle 1996) avance certaines options d'appui demandées à la fois par les clientes des services communautaires et la population en général d'Ottawa-Carleton ayant un revenu de moins de $30000 \$$ : des comptoirs alimentaires, des comptoirs de vêtements, des cuisines collectives, une maison communautaire, des soupes populaires, du soutien à domicile. Les programmes d'aide après l'école, la prévention du décrochage scolaire, le counselling familial et les groupes de parentage, d'entraide et d'appui semblent aussi importants à signaler. Mais que restera-t-il après les coupures de ces appuis aux familles? Qu'adviendra-t-il de programmes de promotion communautaire, comme «Partir d'un bon pas pour un avenir meilleur» (Peters 1994)? Ces programmes offrent l'occasion d'apprentissage d'habiletés sociales et parentales; mais surtout, ils peuvent être des lieux et des milieux privilégiés pour qu'émergent la participation communautaire et les sentiments collectifs.

L'empowerment appliqué à l'intervention familiale individualisée perd vraiment une partie fondamentale de son sens s'il ne débouche pas sur de telles activités collectives d'entraide, une participation communautaire et des solidarités (Perkins 1995). Pourtant, «il semble que certains contextes et certaines approches réussissent mieux que d'autres à aider ces familles, à les accompagner dans leurs démarches d'autonomie ou d'interdépendance» (St-Amand et al. 1996: 1), voire d'empowerment collectif. La démarche de prise de conscience sociale et de contextualisation, ici amorcée en intervention individualisée, s'avère cruciale. StAmand et al. insistent aussi sur «la création d'un espace de solidarité, d'un endroit où l'on se sent chez soi (...), sur la sensibilité et l'extrême attention apportée aux dimensions culturelles et sociales de la vie familiale au niveau local, sur la participation des familles aux organismes alternatifs» (1996: 6). Il y a là un vibrant appel à l'écoute des ressources de ces familles, au respect de leur culture et à une voie alternative.

Au contraire, une perspective restrictive de l'empowerment des familles, sans analyse critique, ni contextualisation ancrée des 
"Outre le potentiel des milieux et l'entraide possible dans une communauté, où sont les ressources mobilisables pour les familles pauvres dans un contexte de carence, d'appauvrissement et de coupures généralisées des services d'appui aux familles, de coupures massives à l'aide sociale, de précarisation d'emplois et de workfare?» difficultés individuelles et familiales va rapidement dans le sillon d'une définition technocratique de l'empowerment. La prise en charge, l'adaptation sociale et l'appui uniquement sur des ressources informelles de soutien, ne sont-ils pas des voies intéressantes pour la politique ontarienne minimaliste en matière de support familial et d'intervention sociale? Qu'adviendra-t-il de l'intervention sur des conflits internes majeurs dans les familles, sur les hauts risques dégénérant en violence, en protection, déviance et placement? Assurera-t-on alors la sécurité des enfants, des familles et du public? Outre le potentiel des milieux et l'entraide possible dans une communauté, où sont les ressources mobilisables pour les familles pauvres dans un contexte de carence, d'appauvrissement et de coupures généralisées des services d'appui aux familles, de coupures massives à l'aide sociale, de précarisation d'emplois et de workfare? N'est-ce pas une sur-responsabilisation individuelle d'accès à des ressources limitées, et ce sans aucune analyse sociale? Au nom de l'empowerment et de sa prise en charge, on occulterait une surresponsabilisation individuelle des problèmes. Ce concept permettrait de nier la pauvreté et son impact sur des individus et des familles, car la pauvreté est ainsi mise sous silence derrière la notion de hauts risques.

De surcroit, au sein de ce débat social de fond sur la pauvreté et la perte d'acquis sociaux, il ne faudra pas perdre de vue la survie des services sociaux en français. Les services spécifiques d'investissement auprès des enfants, des jeunes et des familles francophones, incluant les familles vivant dans la pauvreté, serontils sacrifiés pour sauvegarder quelques services bilingues? Les familles pauvres francophones certes doublement marginalisées tireraient avantage à une démarche d'empowerment, dans toute la vigueur de sa définition. Ce concept ne doit surtout pas devenir un terme banalisé, utilisé abusivement, laissant place à une perspective technocratique de prise en charge, qui ouvre la porte à des coupures et qui les justifie. Pourtant, l'empowerment en soi contredit cette perspective; même sous un angle uniquement psychologique, l'empowerment contrecarre ces abus. 


\section{Bibliographie}

ADAMS, Robert (1996). Social Work and Empowerment, London, MacMillan Press.

ANDREAE, Dan (1996). «Message du président: les travailleurs sociaux en progrès malgré une conjoncture difficile", Bulletin: la revue de l'Association des travailleuses et travailleurs sociaux de l'Ontario, vol. 23, no 3, 3-4.

ASSOCIATION ONTARIENNE DES CENTRES DE SANTÉ MENTALE POUR ENFANTS (1996). Mémo, décembre.

BOUDREAULT, Jean et al. (1994). Vers un continuum de services intégrés à la jeunesse: les solutions de rechange au placement des jeunes - Rapport du Comité consultatif sur le développement de solutions de rechange en matière de placements d'enfants, Québec, ministère de la Santé et des services sociaux.

BRETON, Margot (1994a). On the Meaning of Empowerment and Empowerment - Oriented Social Work Practice, Social Work with Groups, vol. 17, no 3, 23-37.

BRETON, Margot (1994b). Relating Competence - Promotion and Empowerment, Journal of Progressive Human Services, vol. 5, no 1, 27-44.

CARRIER, Gaby, Marie-Christine SAINT-JACQUES, Laurent CHABOT et MichelTHIBAULT (1992). «Les services intensifs : une nouvelle approche dans l'intervention auprès des familles à risque», Service social, vol. 41, no 3, 41-75.

CENTRE PSYCHO-SOCIAL POUR ENFANTS ET FAMILLES (1996). Mémo descriptif de la clientèle, janvier 1997.

COLE, Elizabeth S. (1995). Becoming Family Centered : Child Welfare's Challenge, Families in Society: The Journal of Contemporary Human Services, vol. 76, no 3, 163-172.

CONSEIL NATIONAL DU BIEN-ÊTRE SOCIAL (1996). Profil de la pauvreté, 1994 - Rapport, Ottawa, Ministère des Approvisionnements et Services Canada.

DAGENAIS, Christian et Camil BOUCHARD (1993). «Intervention massive ou intervention magique? Les programmes de soutien intensif aux familles», P.R.I.S.M.E., vol. 3, no 4, 503-515.

DAGENAIS, Huguette (1985). "Les femmes et le pouvoir», dans Colette Gendron et Micheline Beauregard, eds. Les femmes et la santé, Chicoutimi, Éditions Gaëtan Morin, 107-118.

DALLAIRE, Nicole et Claire CHAMBERLAND. "Empowerment, crises et modernité», Revue canadienne de santé mentale communautaire, article à paraître.

DIALLO, Lamine et Marge REITSMA-STREET (1995). «Stratégies de survie et d'identité : les dynamiques culturelles dans un projet d'intervention en prévention communautaire», Reflets: revue ontaroise d'intervention sociale et communautaire, vol. 1, no 1, 43-69.

DORE, Martha M. (1993). Family Preservation and Poor Families: When Homebuilding Is Not Enough, Families in Society: The Journal of Contemporary Human Services, vol. 74, no 9, 544-556.

DUBOIS, Brenda et Karla KROGSRUD MILEY (1996). Social Work: An Empowering Profession, Needham Heights, Allyn and Bacon.

DUNST, Carl J., Carol M.TRIVETTE et Angela G. DEAL (eds.) (1994). Supporting EStrengthening Families - Volume 1: Methods, Strategies and Practices, Cambridge, Brookline Books.

FETTERMAN, David M., Shakeh J. KAFTARIAN et Abraham WANDERSMAN (eds.) (1996). Empowerment Evaluation: Knowledge and Tools for Self-Assessment \& Accountability, Thousand Oaks, Sage Publications. 
FRIEDMANN, John (1996). Rethinking Poverty: Empowerment and Citizen Rights, International Social Science Journal, vol. 148, 161-172.

GARBARINO, James, Cynthia J. SCHELLENBACH, Janet M. SEBES et al. (1986). Troubled Youth, Troubled Families: Understanding At-Risk for Adolescent Maltreatment, New York, Aldine de Gruyter.

GELLES, Richard J. (1993). Through a Sociological Lens: Social Structure and FamilyViolence, dans Richard J. Gelles et Donileen R. Loseke, eds. Current Controversies on Family Violence, Newbury Park, Sage Publications, 31-46.

GINGRAS, Pauline (1992). Le traitement en première ligne des demandes individuelles d'aide en CLSC selon une approche communautaire: guide de référence, Québec, Fédération des CLSC.

GUTIÉRREZ, Lorraine M. (1994). Understanding the Empowerment Process:Does Consciousness Make a Difference?, Social Work Research, vol. 19, no 4, 229-237.

GUTTIÉRREZ, Lorraine, Kathryn A. DeLOIS et Linnea GLENMAYE (1995). Understanding Empowerment Practice: Building on Practitioner-Based Knowledge, Families in Society: The Journal of Contemporary Human Sciences, vol. 76, no 7, 534-544.

HODGES,Vanessa G. et Betty J. BLYTHE (1992). Improving Service Delivery to High-Risk Families: Home-Based Practice, Families in Society: The Journal of Contemporary Human Services, vol. 73, no $5,259-265$.

KAPLAN, Lisa et Judith L. GIRARD (1994). Strengthening High-Risk Families: A Handbook for Practitioners, New York, Lexington Books.

KIEFFER, Charles H. (1984). Citizen Empowerment: A Developmental Perspective, Prevention in Human Services, vol. 3, no 2-3, 9-36.

KINNEY,Jill,James K.WHITTAKER, Elizabeth M.TRACY et Charlotte BOOTH (1990). Reaching High-Risk Families: Intensive Family Preservation in Human Services, New York, Aldine de Gruyter.

KISSMAN, Kris et Jo Ann ALLEN (1993). Single-Parent Families, Newbury Park, Sage Publications.

LE BOSSÉ,Yann. (1996). «Empowerment et pratiques sociales : illustration du potentiel d'une utopie prise au sérieux", Nouvelles pratiques sociales, vol. 9, no 1,127-147.

LE BOSSÉ,Yann (1995). Étude exploratoire du phénomène de l'Empowerment, thèse de doctorat déposée à la Faculté des études graduées de l'Université Laval, Québec.

LE BOSSÉ,Yann D. et Marguerite LAVALLÉE (1993). «Empowerment et psychologie communautaire: aperçu historique et perspectives d'avenir», Les cahiers internationaux de psychologie sociale, no.18, $7-20$.

LEE, Judith A. B. (1994). The Empowerment Approach to Social Work Practice, New York, Columbia University Press.

LÉVESQUE, Justin et Jean PANET-RAYMOND (1994). «L'évolution et la pertinence de l'approche structurelle dans le contexte social actuel», Service social, vol. 43, no 3, 23-39.

MALENFANT, Brigitte et Louise DELISLE (1996). Les services communautaires: une étude des besoins francophones d'Ottawa-Carleton - Rapport de projet, Ottawa, Conseil de planification sociale d'Ottawa-Carleton.

MOREAU, Maurice et al. (1993). Empowerment II: Snapshots of the Structural Approach in Action, Ottawa, Librairie nationale du Canada.

MOREAU, Maurice et Lynne LEONARD (1989). Empowerment Through a Structural Approach to Social Work: A Report from Practice, Ottawa, Carleton University. 
MULLENDER, Audrey et David WARD (1991). Empowerment Through Social Action Group Work: 'The Self-Directed' Approach, Social Work with Groups, vol. 14, no 3-4, 125-139.

NINACS, William A. (1995). Empowerment et service social: approches et enjeux, Service social, vol. 44, no 1, 69-93.

ODESSE, Monique (1991). Programmes d'intervention auprès des familles d'enfants placés en centre d'accueil: une recension d'écrits, Québec, Université Laval.

OZER, Elizabeth M. et Albert BANDURA (1990). Mechanisms Governing Empowerment Effects: A Self-Efficacy Analysis, Journal of Personality and Social Psychology, vol. 58, no 3, 472-486.

PARSLEO, Phyllida (1995). The Concept of Empowerment in Social Work Practice, Hong Kong Journal of Social Work, vol. 29, no1, pp 1-11.

PARSONS, Ruth J. (1991). Empowerment: Purpose and Practice Principle in Social Work, Social Work with Groups, vol. 14, no 2, 7-21.

PERKINS, Douglas D. (1995). Speaking Truth to Power: Empowerment Ideology as Social Intervention and Policy, American Journal of Community Psychology, vol. 23, no. 5, 765-793.

PERKINS, Douglas D. et Marc A. ZIMMERMAN (1995). Empowerment Theory, Research, and Application, American Journal of Community Psychology, vol. 23, no 5, 569-579.

PETERS, Ray DeV. (1994). Better Beginnings, Better Futures: A Community-Based Approach to Primary Prevention, Canadian Journal of Community Mental Health, vo. 13, no 2, 183-188.

PINDERHUGHES, Elaine (1995). Empowering Diverse Populations: Family Practice in the 21st Century, Families in Society: The Journal of Contemporary Human Services, vol. 76, no 3, 131-140.

RAPPAPORT, Julian (1995). Empowerment Meets Narrative: Listening to Stories and Creating Settings, American Journal of Community Psychology, vol. 23, no 5, 795-807.

RAPPAPORT, Julian (1993). Narrative Studies, Personal Stories, and Identity in Mutual Help Context, The Journal of Applied Behavioral Science, vol. 29, no 2, 239-256.

RAPPAPORT, Julian (1987). Terms of Empowerment/Exemplars of Prevention: Toward a Theory for Community Psychology, American Journal of Community Psychology, vol.15, no.2, 121-148.

RÉGIE RÉGIONALE DE LA SANTÉ ET DES SERVICES SOCIAUX (MAURICIE-BOISFRANCS) (1996). La transformation du réseau de la santé et des services sociaux en Mauricie-BoisFrancs: Recommandations 1996, Trois-Rivières, Régie régionale de la santé et des services sociaux (Mauricie-Bois-Francs).

RONNAU, John P. et Christine R. MARLOW (1993). Family Preservation, Poverty, and the Value of Diversity, Families in Society: The Journal of Contemporary Human Services, vol. 74, no 9, 538-544.

ST-AMAND, Nérée et al. (1996). Ici, c’est chez nous : ressources alternatives pour familles démunies, Ottawa, École de service social de l'Université d'Ottawa.

SHIELDS, Laurence E. (1995).Women's Experiences of the Meaning of Empowerment, Qualitative Health Research, vol. 5, no 1,15-35.

SIMARD, Juliette et Daniel TURCOTTE (1992). La thérapie orientée vers la solution: un modèle applicable à l'intervention en contexte d'autorité, Service social, vol. 41, no 3, 77-93.

SIMON, Barbara Levy (1994). The Empowerment Tradition in American Social Work: A History, New York, Columbia University Press.

TOURIGNY, Marc, Christian DAGENAIS, Julie TURNER et Léna LORTIE (1995). «Évaluation 
de l'implantation du projet d'intervention massive à l'enfance (prime) pour les deux premières années, 93-94 et 94-95», GRAVE: Groupe de recherche et d'action sur la victimisation des enfants, vol. 2, no 6.

TROCMÉ, Nico (1994). «Le rôle des facteurs de classe et de genre dans la sélection de stratégies de recherche, d'intervention et de prévention de la maltraitance des enfants», dans Francine Ouellet et Michèle Clément eds. Violences dans les relations affectives : représentations et interventions, Québec, CRI-VIFF, 7-33.

TROCMÉ, Nico, Debra McPHEE et Kwok KWAN TAM (1995). Child Abuse and Neglect in Ontario: Incidence and Characteristics, Child Welfare, vol. 74, no 3, 563-586.

VINCENT, Carol (1996). Parent Empowerment? Collective Action and Inaction in Education, Oxford Review of Education, vol. 22, no 4, pp 465-482.

VITARO, Frank, Patricia L. DOBKIN, Claude GAGNON et Marc LEBLANC (1994). Les problèmes d'adaptation psychosociale chez l'enfant et l'adolescent: prévalence, déterminants et prévention, Sainte-Foy, Presses de l'Université du Québec.

ZIMMERMAN, Marc A. (1995). Psychological Empowerment: Issues and Illustrations, American Journal of Community Psychology, vol. 23, no 5, 581-599.

ZIMMERMAN, Marc A. (1990). Taking Aim on Empowerment Research: On the Distinction Between Individual and Psychological Conceptions, American Journal of Community Psychology, vol. 18, no 1, 169-177.

ZIMMERMAN, Marc A., Barbara A. ISRAEL, Amy SCHULZ et Barry CHECKOWAY (1992). Further Explorations in Empowerment Theory: An Empirical Analysis of Psychological Empowerment, American Journal of Community Psychology, vol. 20, no 6, 707-727.

ZIMMERMAN, Marc A. et Julian RAPPAPORT (1988). Citizen Participation, Perceived Control, and Psychological Empowerment, American Journal of Community Psychology, vol. 16, no 5, 725-750.

1. Nous tenons à remercier pour leurs commentaires judicieux mesdames Marthe Duhamel-Maples et Angèle Bouchard, ainsi que monsieur Charles Lachance du Centre psycho-social pour enfants et familles d'Ottawa-Carleton. La précieuse collaboration de monsieur Alain Charpentier, professeur à la Cité Collégiale d'Ottawa, fut des plus appréciées. 